\title{
Neutron single spin asymmetries from semi-inclusive deep inelastic scattering off transversely polarized ${ }^{3} \mathrm{He}$
}

\author{
Sergio Scopetta* \\ Dipartimento di Fisica, Università degli Studi di Perugia, and INFN, sezione di Perugia via A. Pascoli 06100 Perugia, Italy, \\ and Departament de Fisica Teòrica, Universitat de València, 46100 Burjassot (València), Spain
}

(Received 28 December 2006; published 6 March 2007)

\begin{abstract}
A study of semi-inclusive deep inelastic scattering off transversely polarized ${ }^{3} \mathrm{He}$ is presented. The formal expressions of the Collins and Sivers contributions to the azimuthal single spin asymmetry for the production of leading pions are derived, in impulse approximation, and estimated in the kinematics of forth-coming experiments at JLab. The AV18 interaction has been used for a realistic description of the nuclear dynamics; the nucleon structure has been described by proper parametrizations of data or suitable model calculations. The initial transverse momentum of the struck quark has been properly included in the calculation. The crucial issue of extracting the neutron information from ${ }^{3} \mathrm{He}$ data, planned to shed some light on the puzzling experimental scenario arisen from recent measurements for the proton and the deuteron, is thoroughly discussed. It is found that a model independent procedure, widely used in inclusive deep inelastic scattering to take into account the momentum and energy distributions of the bound nucleons in ${ }^{3} \mathrm{He}$, can be applied also in the kinematics of the planned JLab experiments, although fragmentation functions, not only parton distributions, are involved. The possible role played by final state interactions in the process under investigation is addressed.
\end{abstract}

DOI: 10.1103/PhysRevD.75.054005

\section{INTRODUCTION}

The distribution of transverse quark spin, the so-called transversity distribution, is one of the least known features of the nucleon partonic structure (for a recent review, see, e.g., Ref. [1]). In a few years, some light should be shed on it, thanks to relevant experimental efforts which are in turn stimulating the theoretical activity (for recent developments, see Ref. [2]).

Semi-inclusive deep inelastic scattering (SIDIS), i.e. the process $A\left(e, e^{\prime} h\right) X$, with the detection in the final state of a produced hadron $h$ in coincidence with the scattered electron $e^{\prime}$, is one of the proposed processes to access the transversity distribution, $h_{1}$, and other related observables. In fact, due to its chiral-odd nature, $h_{1}$ is not observable in inclusive deep inelastic scattering (DIS), while it can appear, multiplied by a chiral-odd fragmentation function, in the expression of SIDIS cross-sections. For several years it has been known that SIDIS off a transversely polarized target should show azimuthal asymmetries, the so-called "single spin asymmetries" (SSAs). As a matter of fact, it is predicted that the counting of produced hadrons in a given direction or in the opposite one, with respect to the reaction plane, depends on the orientation of the transverse spin of a polarized target with respect to the direction of the unpolarized beam. It can be shown that the SSA in SIDIS off transverse polarized targets is essentially due to two different physical mechanisms. One of them is the Collins mechanism, due to parton final state interactions in the production of a spinless hadron by a transversely polarized quark. This mechanism produces a term in the SSA given

*Electronic address: sergio.scopetta@pg.infn.it
PACS numbers: $13.60 . \mathrm{Hb}, 13.85 . \mathrm{Hd}, 13.85 . \mathrm{Ni}, 13.87 . \mathrm{Fh}$

essentially by the product of the transversity distribution and the Collins fragmentation function. This latter quantity is time reversal odd (T-odd) and counts the number density of scalar hadrons originating from the fragmentation of a transversely polarized quark [3]. The other is the Sivers mechanism, producing a term in the SSA which is given by the product of the unpolarized fragmentation function with the Sivers parton distribution [4], naturally related to the parton Orbital Angular Momentum (OAM), describing the number density of unpolarized quarks in a transversely polarized target.

It turns out that, in the experimental asymmetry, it is technically possible to distinguish the different contributions of the Collins and Sivers mechanisms [5-7].

Recently, the first data of SIDIS off transverse polarized targets have been published, for the proton [8] and the deuteron [9]. It has been found that, while the Sivers effect is sizable for the proton, it becomes negligible for the deuteron, so that apparently the neutron contribution cancels the proton one, showing a strong flavor dependence of the mechanism. Very recently, it has been argued that these results can be interpreted as an evidence of a negligible gluon contribution to the OAM of the partons in the proton [10]. In the same paper, it is also stressed that a transversely polarized ${ }^{3} \mathrm{He}$ target, due to its peculiar spin structure $[11,12]$, could be used to obtain relevant information on SSAs of the free neutron, to better understand the present experimental scenario.

With the aim at measuring the neutron transversity and the neutron SSAs, two experiments of SIDIS off transversely polarized ${ }^{3} \mathrm{He}$ targets have been approved at JLab, with scientific rate "A", for the detection of $\pi^{-}$ (experiment E-06-010 [13]) and $\pi^{+}$(experiment E-06- 
011 [14]) in coincidence with the scattered electron, in the valence quark region $(0.19 \leq x \leq 0.41)$, for moderate values of $Q^{2}\left(1.77 \mathrm{GeV}^{2} \leq Q^{2} \leq 2.73 \mathrm{GeV}^{2}\right)$. It has been planned to detect the leading fragmentation pion which carries $z \simeq 0.5$ of the energy transfer, to favor current fragmentation.

In this theoretical and experimental scenario, it becomes urgent to confirm the possibility to use a transversely polarized ${ }^{3} \mathrm{He}$ target to extract the information for the free neutron. In the present paper, this issue is thoroughly investigated. A calculation of SSAs for SIDIS off transversely polarized ${ }^{3} \mathrm{He}$, described in an impulse approximation (IA) framework, by means of wave functions obtained using a modern nucleon-nucleon potential, will be illustrated. In the calculation, the treatment of the nuclear structure is realistic, while the nucleon part relies on model estimates, being some ingredients of the calculation experimentally unknown. As a consequence, the main goal of the paper is not that of producing realistic predictions, but that of establishing to what extent a transversely polarized ${ }^{3} \mathrm{He}$ nucleus behaves as an effective transversely polarized free neutron.

The paper is structured as follows. In the Sec. II, the main quantities of interest will be introduced. In Sec. III, the IA expressions for the Collins and Sivers contributions to the nuclear SSA will be derived and the calculation will be set-up, in the kinematics of the JLab experiment. The ingredients to be used will be presented in Sec. IV, together with the numerical results and their discussion. Eventually, conclusions will be drawn in Sec. V.

\section{SIDIS AND SINGLE SPIN ASYMMETRIES}

The quantities to be measured are the Collins, $A_{U T}^{\text {Collins }}$, and Sivers, $A_{U T}^{\text {Sivers }}$, contributions to the azimuthal single spin asymmetry, $A_{U T}$ (where $U$ means Unpolarized beam, $T$ means Transversely polarized target and it is assumed that the produced hadron is spinless, or that its polarization is not detected) [7]

$$
\begin{aligned}
A_{U T}\left(\phi_{h}, \phi_{S}\right) & \equiv \frac{d \sigma\left(\phi_{h}, \phi_{S}\right)-d \sigma\left(\phi_{h}, \phi_{S}+\pi\right)}{d \sigma\left(\phi_{h}, \phi_{S}\right)+d \sigma\left(\phi_{h}, \phi_{S}+\pi\right)} \\
& \equiv \frac{d^{6} \sigma_{U T}}{d^{6} \sigma_{U U}},
\end{aligned}
$$

where the angles are defined according to the "Trento convention" [15], see Fig. 1. The cross sections are differential in $x, y, z, \phi_{S}, \mathbf{h}_{\perp}$, where, as usual, one has

$$
\begin{gathered}
x=\frac{Q^{2}}{2 P \cdot q}, \\
y=\frac{P \cdot q}{P \cdot l}=\frac{\nu}{E_{\mathrm{lab}}}, \\
z=\frac{P \cdot h}{P \cdot q}=\frac{E_{h}^{\mathrm{lab}}}{\nu},
\end{gathered}
$$

where $P, q, h$ are the target momentum, the momentum transfer and the momentum of the produced hadron, respectively. A clarification concerning the notation in SIDIS is in order. In general, a quantity which is transverse in a frame where $P$ and $h$ have no transverse components (thus $\mathbf{P}_{\mathbf{T}}=\mathbf{h}_{\mathbf{T}}=0$ ), is indicated with a subscript $T$, so that $T$ means transverse with respect to $\vec{h}$, while those with a subscript $\perp$ are defined in a frame where $q$ and $P$ have no transverse components (standard DIS frame), so that $\perp$ means transverse with respect to $\vec{q}$ (thus $\mathbf{q}_{\perp}=\mathbf{P}_{\perp}=\mathbf{0}$ ) [5].

Other relevant variables for the description of this process are $\mathbf{k}_{\mathbf{T}}$, the transverse momentum of the struck quark

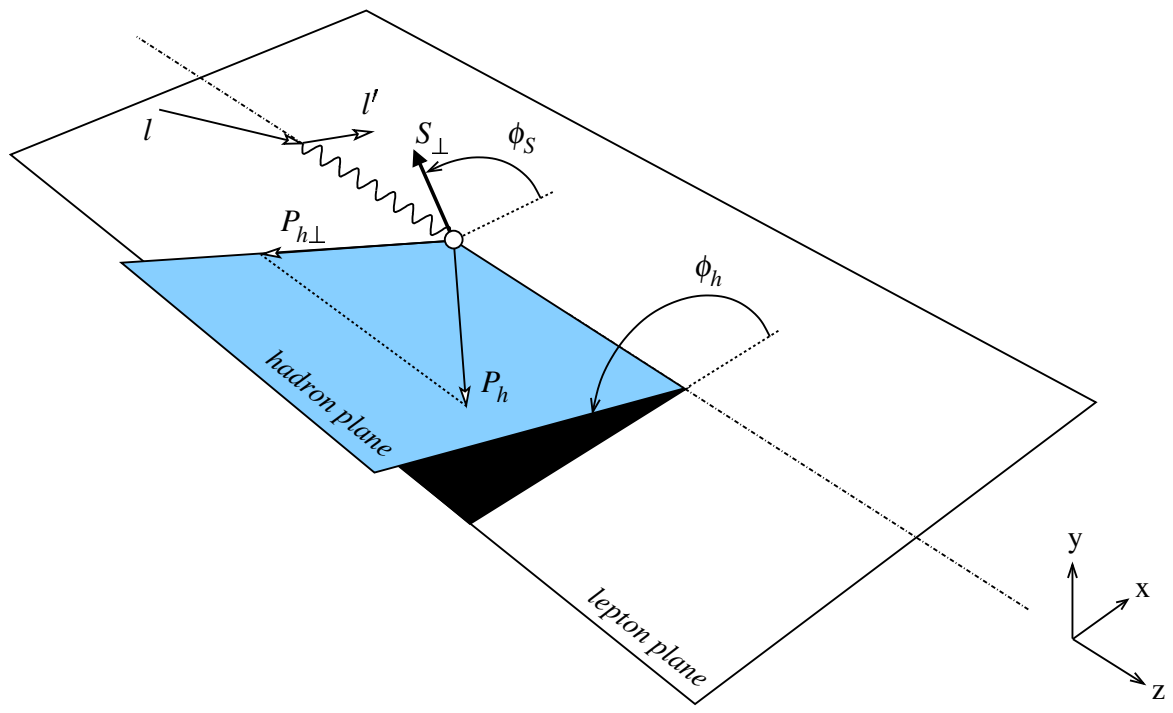

FIG. 1 (color online). Definition of the azimuthal angles for the process $A \uparrow\left(e, e^{\prime} h\right) X$, in the rest frame of the target $A$, according to the "Trento convention" (after Ref. [15]). 
before the interaction, and $\kappa_{\mathrm{T}}$, the transverse momentum of the struck quark after the interaction. It is found that $\mathbf{q}_{\mathbf{T}}=$ $-\mathbf{h}_{\perp} / z+O\left(1 / Q^{2}\right)$.

The Collins and Sivers asymmetries, according to the Trento convention [15], are defined in terms of the experimental cross sections

$$
A_{U T}^{\text {Collins }}=\frac{\int d \phi_{S} d \phi_{h} \sin \left(\phi_{h}+\phi_{s}\right) d^{6} \sigma_{U T}}{\int d \phi_{S} d \phi_{h} d^{6} \sigma_{U U}},
$$

$$
A_{U T}^{\text {Sivers }}=\frac{\int d \phi_{S} d \phi_{h} \sin \left(\phi_{h}-\phi_{S}\right) d^{6} \sigma_{U T}}{\int d \phi_{S} d \phi_{h} d^{6} \sigma_{U U}} .
$$

The cross sections themselves can be written in terms of $\mathbf{k}_{\mathbf{T}}$-dependent distribution and fragmentation functions [5], so that also the Collins and Sivers asymmetries, Eqs. (5) and (6), can be written through the same functions. In particular, $A_{U T}^{\text {Collins }}$ is found to be

$$
A_{U T}^{\text {Collins }}=\frac{1-y}{1-y+y^{2} / 2}\left|\mathbf{S}_{\mathbf{T}}\right| \frac{\sum_{q} e_{q}^{2} \int d \phi_{S} d \phi_{h} d^{2} \kappa_{\mathbf{T}} d^{2} \mathbf{k}_{\mathbf{T}} \delta^{2}\left(\mathbf{k}_{\mathbf{T}}+\mathbf{q}_{\mathbf{T}}-\kappa_{\mathbf{T}}\right) \frac{\hat{\mathbf{h}} \kappa_{\mathbf{T}}}{\mathbf{M}_{\mathbf{h}}} h_{1}^{q}\left(x, \mathbf{k}_{\mathbf{T}}^{\mathbf{2}}\right) H_{1}^{\perp q, h}\left(z,\left(z \kappa_{\mathbf{T}}\right)^{\mathbf{2}}\right)}{\sum_{q} e_{q}^{2} \int d \phi_{S} d \phi_{h} d^{2} \kappa_{\mathbf{T}} d^{2} \mathbf{k}_{\mathbf{T}} \delta^{2}\left(\mathbf{k}_{\mathbf{T}}+\mathbf{q}_{\mathbf{T}}-\kappa_{\mathbf{T}}\right) f_{1}^{q}\left(x, \mathbf{k}_{\mathbf{T}}^{\mathbf{2}}\right) D_{1}^{q, h}\left(z,\left(z \kappa_{\mathbf{T}}\right)^{\mathbf{2}}\right)},
$$

where $\mathbf{S}_{\mathbf{T}}$ is the transverse spin of the target hadron, $\hat{\mathbf{h}}_{\perp}=\mathbf{h}_{\perp} /\left|\mathbf{h}_{\perp}\right|, M_{h}$ is the mass of the produced hadron and two $\mathbf{k}_{\mathbf{T}}$-dependent distributions, the unpolarized one, $f_{1}^{q}\left(x, \mathbf{k}_{\mathbf{T}}^{\mathbf{2}}\right)$, and the transversity distribution, $h_{1}^{q}\left(x, \mathbf{k}_{\mathbf{T}}^{\mathbf{2}}\right)$, appear. The other two ingredients of Eq. (7) are the $\mathbf{k}_{\mathbf{T}}$-dependent unpolarized fragmentation function for the production of a scalar hadron, $D_{1}^{q, h}\left(z,\left(z \kappa_{\mathbf{T}}\right)^{2}\right)$, and the quantity $H_{1}^{\perp,, h}\left(z,\left(z \kappa_{\mathbf{T}}\right)^{2}\right)$, the so-called Collins fragmentation function, a T-odd quantity describing the number density of scalar hadrons originating from the fragmentation of a transversely polarized quark [3].

In the same way, $A_{U T}^{\text {Sivers }}$ is found to be

$$
A_{U T}^{\text {Sivers }}=\left|\mathbf{S}_{\mathbf{T}}\right| \frac{\sum_{q} e_{q}^{2} \int d \phi_{S} d \phi_{h} d^{2} \kappa_{\mathbf{T}} d^{2} \mathbf{k}_{\mathbf{T}} \delta^{2}\left(\mathbf{k}_{\mathbf{T}}+\mathbf{q}_{\mathbf{T}}-\kappa_{\mathbf{T}}\right) \frac{\hat{\mathbf{h}} \cdot \mathbf{k}_{\mathbf{T}}}{\mathbf{M}} f_{1 T}^{\perp q}\left(x, \mathbf{k}_{\mathbf{T}}^{\mathbf{2}}\right) D_{1}^{q, h}\left(z,\left(z \kappa_{\mathbf{T}}\right)^{2}\right)}{\sum_{q} e_{q}^{2} \int d \phi_{S} d \phi_{h} d^{2} \kappa_{\mathbf{T}} d^{2} \mathbf{k}_{\mathbf{T}} \delta^{2}\left(\mathbf{k}_{\mathbf{T}}+\mathbf{q}_{\mathbf{T}}-\kappa_{\mathbf{T}}\right) f_{1}^{q}\left(x, \mathbf{k}_{\mathbf{T}}^{\mathbf{2}}\right) D_{1}^{q, h}\left(z,\left(z \kappa_{\mathbf{T}}\right)^{\mathbf{2}}\right)},
$$

where $M$ is the target mass and, with respect to the previous asymmetry, Eq. (7), there is a new $\mathbf{k}_{\mathbf{T}}$-dependent distribution function, $f_{1 T}^{\perp q}\left(x, \mathbf{k}_{\mathbf{T}}^{\mathbf{2}}\right)$. The latter is a T-odd quantity, the so-called Sivers distribution function, describing the number density of unpolarized quarks in a transversely polarized target [4].

Often, an $x-z$ factorized structure is assumed for Eqs. (7) and (8), a procedure which is justified if there is a good experimental coverage in $\left|\mathbf{h}_{\perp}\right|$. In fact, in this case, one can integrate the numerator and the denominator of Eq. (1) over $\mathbf{h}_{\perp}=-z \mathbf{q}_{\mathbf{T}}$, with proper weights, selecting the Collins and Sivers contributions and getting simplified expressions for the azimuthal asymmetries [6,7]. Because of the peculiar kinematical conditions of the JLab experiments $[13,14]$, where $\left|\mathbf{h}_{\perp}\right|$ is varying in a narrow range, such a procedure will not be applied here. The general expressions, Eqs. (7) and (8), for the Collins and Sivers asymmetries, will be considered in what follows.

\section{AN IMPULSE APPROXIMATION APPROACH TO SIDIS OFF TRANSVERSELY POLARIZED ${ }^{3} \mathrm{He}$}

An Impulse Approximation (IA) approach to perform the first calculation of the Collins and Sivers Asymmetries of ${ }^{3} \mathrm{He}$ will be now described. The idea is that in the SIDIS process a single nucleon is interacting with the hard probe and there are no further interactions with the recoiling nuclear system, neither of the jet originating from the interacting nucleon, nor of the emitted hadron. The other crucial assumption of IA is that the internal structure of the bound nucleon is not different from that of the free one, the nuclear dynamics determining only its momentum and its binding energy distributions.

Now it will be shown that, in this framework, the Collins and Sivers Asymmetries of ${ }^{3} \mathrm{He}$, for the production of a hadron $h$, in the kinematics of the JLab experiments, are given by the following convolution expressions:

$$
A_{U T, h}^{\text {Collins, }{ }^{3} \mathrm{He}} \simeq \frac{1-y}{1-y+y^{2} / 2}\left|\mathbf{S}_{\mathbf{T}}\right| \frac{N^{\text {Collins, }{ }^{3} \mathrm{He}}}{D^{3} \mathrm{He}}
$$

and

$$
A_{U T, h}^{\text {Sivers, }{ }^{3} \mathrm{He}} \simeq\left|\mathbf{S}_{\mathbf{T}}\right| \frac{N^{\mathrm{Sivers},{ }^{3} \mathrm{He}}}{D^{3 \mathrm{He}}},
$$

where:

$$
\begin{aligned}
N^{\text {Collins, }{ }^{3} \mathrm{He}}= & \sum_{N=n, p} \sum_{q} e_{q}^{2} \int d \phi_{S} d \phi_{h} d^{2} \kappa_{\mathbf{T}} d^{2} \mathbf{k}_{\mathbf{T}} \\
& \times \delta^{2}\left(\mathbf{k}_{\mathbf{T}}+\mathbf{q}_{\mathbf{T}}-\kappa_{\mathbf{T}}\right) \frac{\hat{\mathbf{h}} \cdot \kappa_{\mathbf{T}}}{\mathbf{M}_{\mathbf{h}}} H_{1}^{\perp q, h}\left(z,\left(z \kappa_{\mathbf{T}}\right)^{\mathbf{2}}\right) \\
& \times \int_{x}^{M_{3} / M} \frac{d \alpha}{\alpha} G_{\perp}^{3, N}(\alpha) h_{1}^{q, N}\left(\frac{x}{\alpha}, \mathbf{k}_{\mathbf{T}}^{2}\right)
\end{aligned}
$$




$$
\begin{aligned}
D^{3} \mathrm{He}= & \sum_{N=n, p} \sum_{q} e_{q}^{2} \int d \phi_{S} d \phi_{h} d^{2} \kappa_{\mathbf{T}} d^{2} \mathbf{k}_{\mathbf{T}} \\
& \times \delta^{2}\left(\mathbf{k}_{\mathbf{T}}+\mathbf{q}_{\mathbf{T}}-\kappa_{\mathbf{T}}\right) D_{1}^{q, h}\left(z,\left(z \kappa_{\mathbf{T}}\right)^{2}\right) \\
& \times \int_{x}^{M_{3} / M} \frac{d \alpha}{\alpha} F^{3, N}(\alpha) f_{1}^{q, N}\left(\frac{x}{\alpha}, \mathbf{k}_{\mathbf{T}}^{\mathbf{2}}\right),
\end{aligned}
$$

and

$$
\begin{aligned}
N^{\text {Sivers, }{ }^{3} \mathrm{He}}= & \sum_{N=n, p} \sum_{q} e_{q}^{2} \int d \phi_{S} d \phi_{h} d^{2} \kappa_{\mathbf{T}} d^{2} \mathbf{k}_{\mathbf{T}} \\
& \times \delta^{2}\left(\mathbf{k}_{\mathbf{T}}+\mathbf{q}_{\mathbf{T}}-\kappa_{\mathbf{T}}\right) \frac{\hat{\mathbf{h}} \cdot \mathbf{k}_{\mathbf{T}}}{\mathbf{M}} D_{1}^{q, h}\left(z,\left(z \kappa_{\mathbf{T}}\right)^{\mathbf{2}}\right) \\
& \times \int_{x}^{M_{3} / M} \frac{d \alpha}{\alpha} G_{\perp}^{3, N}(\alpha) f_{1 T}^{\perp q, N}\left(\frac{x}{\alpha}, \mathbf{k}_{\mathbf{T}}^{\mathbf{2}}\right)
\end{aligned}
$$

Now the quantities appearing in the above equations will be described. First of all, the variable

$$
\alpha=\frac{\sqrt{2} p^{+}}{M}=\frac{p_{0}+p_{3}}{M}
$$

represents the light-cone plus-momentum component of the nucleon $N$, with momentum $p$, to which the struck quark belongs, divided by the nucleon mass, $M$ [16].

The functions $G_{\perp}^{3, N}(\alpha)$ and $F^{3, N}(\alpha)$ are the transverse spin-dependent and the spin-independent light-cone momentum distributions of the nucleon $N$ in ${ }^{3} \mathrm{He}$, respectively, defined as

$$
G_{\perp}^{3, N}(\alpha)=\int d E \int d \vec{p} \frac{\sqrt{2} p^{+}}{p_{0}} P_{\perp}^{N}(\vec{p}, E) \delta\left(\alpha-\frac{\sqrt{2} p^{+}}{M}\right),
$$

and

$$
F^{3, N}(\alpha)=\int d E \int d \vec{p} \frac{\sqrt{2} p^{+}}{p_{0}} P^{N}(\vec{p}, E) \delta\left(\alpha-\frac{\sqrt{2} p^{+}}{M}\right) .
$$

Both these quantities depend on the nuclear structure, being the functions

$$
P_{\perp}^{N}(\vec{p}, E)=P_{(1 / 2)(1 / 2),(1 / 2)}^{N}(\vec{p}, E)-P_{-(1 / 2)-(1 / 2),(1 / 2)}^{N}(\vec{p}, E)
$$

and

$$
P^{N}(\vec{p}, E)=P_{(1 / 2)(1 / 2),(1 / 2)}^{N}(\vec{p}, E)+P_{-(1 / 2)-(1 / 2),(1 / 2)}^{N}(\vec{p}, E),
$$

defined in terms of the components of the spin-dependent spectral function of the nucleon $N$ in the ${ }^{3} \mathrm{He}$ nucleus, firstly defined in [17]

$$
\begin{aligned}
P_{\sigma, \sigma^{\prime}, \mathcal{M}_{x}}^{N}(\vec{p}, E)= & \sum_{f_{(A-1)}}\left\langle\vec{p}, \sigma_{x} ; \psi_{f_{(A-1)}} \mid \psi_{J \mathcal{M}_{x}}\right\rangle \\
& \times\left\langle\psi_{J \mathcal{M}_{x}} \mid \psi_{f_{(A-1)}} ; \vec{p}, \sigma_{x}^{\prime}\right\rangle_{N} \\
& \times \delta\left(E-E_{f_{(A-1)}}+E_{A}\right)
\end{aligned}
$$

where $\left|\psi_{J} \mathcal{M}_{x}\right\rangle$ is the ground state of the target nucleus polarized along the $x$ axis, $\left|\psi_{f_{(A-1)}}\right\rangle$ an eigenstate of the $(A-1)$ nucleon system interacting with the same two-body potential of the target nucleus, and $\left|\vec{p}, \sigma_{x}\right\rangle_{N}$ the plane wave state for the nucleon $N$ with the spin projection along the $x$-axis equal to $\sigma_{x}$. From Eqs. (14)-(16) it is clear that, in the extreme nonrelativistic (NR) limit, i.e. with nucleons at rest, $\alpha=1$ and no nuclear effects are found. The size of the leading nuclear effects discussed here is of the order $|\vec{p}| / M$.

One should notice that, in this framework, the probability of finding a transversely polarized nucleon in a transversely polarized nucleus is the same of that of finding a longitudinally polarized nucleon in a longitudinally polarized nucleus, since the only possible treatment of the nuclear system is NR. It turns out therefore that the transverse and longitudinal spin-dependent light-cone momentum distributions are equal and that the results obtained for the latter can be used also for the first quantity, the one of interest here. In other words, the overlaps ${ }_{N}\left\langle\vec{p}, \sigma_{x} ; \psi_{f_{(A-1)}} \mid \psi_{J \mathcal{M}_{x}}\right\rangle$ and ${ }_{N}\left\langle\vec{p}, \sigma_{z} ; \psi_{f_{(A-1)}} \mid \psi_{J \mathcal{M}_{z}}\right\rangle$ turn out to be equal in a NR framework (for the identity of the helicity and transversity distributions in a NR framework see, e.g., Ref. [18,19]).

The main lines of the formal derivation of Eqs. (9) and (10) will be now discussed. It is a generalization of the standard procedure for obtaining the nuclear parton distributions of ${ }^{3} \mathrm{He}$ in DIS. In particular, the derivation of the expression in the numerator of Eqs. (9) and (10) generalizes the one of the ${ }^{3} \mathrm{He}$ helicity distribution, to be found in $[12,20,21]$, while that of the denominator generalizes the I.A. derivation of the ${ }^{3} \mathrm{He}$ unpolarized parton distribution, which can be found, e.g., in Ref. [22]. With respect to the cited papers, the difference here is the presence of the fragmentation functions and of the $\mathbf{k}_{\mathbf{T}}$-dependence in the distribution functions in the SIDIS cross-sections. Let us start from the general expressions Eqs. (7) and (8). In order to evaluate them for a generic $\operatorname{target} A$, one has to estimate therefore three $\mathbf{k}_{\mathbf{T}}$-dependent parton distributions, $f_{1}^{q, A}$,

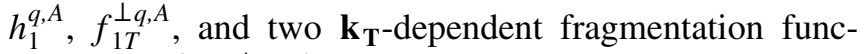
tions, $D_{1}^{q, A, h}, H_{1}^{\perp q, A, h}$. Let us discuss how to obtain the nuclear effects in I.A. for one of the $\mathbf{k}_{\mathbf{T}}$-dependent parton distributions and one of the $\mathbf{k}_{\mathbf{T}}$-dependent fragmentation functions.

As an example, let us consider the parton distribution $f_{1}^{q, A}$, whose definition reads (see, e.g., [1])

$$
\begin{aligned}
f_{1}^{q, A}\left(x, \mathbf{k}_{\mathbf{T}}^{\mathbf{2}}\right)= & \int \frac{d \xi^{-} d \xi_{\mathbf{T}}}{2(2 \pi)^{3}} e^{i\left(x P^{+} \xi^{-}-\mathbf{k}_{\mathbf{T}} \cdot \xi_{\mathbf{T}}\right)} \\
& \times\left\langle P\left|\bar{\Psi}(0,0,0) \gamma^{+} \Psi\left(0, \xi^{-}, \xi_{\mathbf{T}}\right)\right| P\right\rangle,
\end{aligned}
$$

where the light-cone target states $|P\rangle$ are normalized according to

$$
\left\langle P^{\prime} \mid P\right\rangle=(2 \pi)^{3} 2 P^{+} \delta\left(P^{\prime+}-P^{+}\right) \delta^{2}\left(\vec{P}_{\perp}-\vec{P}_{\perp}^{\prime}\right) .
$$


By substituting in Eq. (20) the standard expansion of the quark field $\Psi$, writing for simplicity the quark degrees of freedom only, one gets

$$
\begin{aligned}
f_{1}^{q, A}\left(x, \mathbf{k}_{\mathbf{T}}^{\mathbf{2}}\right)= & \int \frac{d \xi^{-} d \xi_{\mathbf{T}}}{2(2 \pi)^{3}} e^{i\left(x P^{+} \xi^{-}-\mathbf{k}_{\mathbf{T}} \cdot \xi_{\mathbf{T}}\right)} \int \frac{d k^{\prime+} \mathbf{d} \mathbf{k}_{\mathbf{T}}^{\prime}}{2 k^{\prime+}(2 \pi)^{3}} \\
& \times\left\langle P\left|\hat{O} e^{-i\left(k^{\prime+} \xi^{-}-\mathbf{k}_{\mathbf{T}}^{\prime} \cdot \xi_{\mathbf{T}}\right)}\right| P\right\rangle \\
= & \frac{1}{2 P^{+}} \int \frac{d k^{\prime+} \mathbf{d k}_{\mathbf{T}}^{\prime}}{2 k^{\prime+}(2 \pi)^{3}}\langle P|\hat{O}| P\rangle \delta\left(x-\frac{k^{\prime+}}{P^{+}}\right) \\
& \times \delta^{2}\left(\mathbf{k}_{\mathbf{T}}^{\prime}-\mathbf{k}_{\mathbf{T}}\right)
\end{aligned}
$$

where $\hat{O}$ is the quark operator

$$
\hat{O}=\sum_{r} \bar{u}_{r}\left(k^{\prime+}, \mathbf{k}_{\mathbf{T}}^{\prime}\right) b_{r}^{\dagger}\left(k^{\prime+}, \mathbf{k}_{\mathbf{T}}^{\prime}\right) \gamma^{+} b_{r}\left(k^{\prime+}, \mathbf{k}_{\mathbf{T}}^{\prime}\right) u_{r}\left(k^{\prime+}, \mathbf{k}_{\mathbf{T}}^{\prime}\right)
$$

and the creation and annihilation operators, $b^{\dagger}(k)$ and $b(k)$, obey the commutation relation

$$
\left\{b\left(k^{\prime}\right), b^{\dagger}(k)\right\}=(2 \pi)^{3} 2 k^{+} \delta\left(k^{\prime+}-k^{+}\right) \delta^{2}\left(\mathbf{k}_{\mathbf{T}}-\mathbf{k}_{\mathbf{T}}^{\prime}\right) .
$$

Let us think now to a nuclear target with $A$ nucleons. Following a standard procedure, in the above equation two complete sets of states, corresponding to the interacting nucleon in an IA scenario and to the recoiling nuclear system, are properly inserted to the left and right-hand sides of the quark operator

$$
\begin{aligned}
f_{1}^{q, A}\left(x, \mathbf{k}_{\mathbf{T}}^{\mathbf{2}}\right)= & \frac{1}{2 P^{+}} \int \frac{d k^{\prime+} \mathbf{d} \mathbf{k}_{\mathbf{T}}^{\prime}}{2 k^{\prime+}(2 \pi)^{3}}\left\langleP \left|\sum_{\vec{P}_{R}^{\prime}, S_{R}^{\prime}, \vec{p}^{\prime}, s^{\prime}}\left\{\left|\vec{P}_{R}^{\prime} S_{R}^{\prime}\right\rangle\left|\vec{p}^{\prime} s^{\prime}\right\rangle\right\}\right.\right. \\
& \times\left\{\left\langle\vec{P}_{R}^{\prime} S_{R}^{\prime}\left|\left\langle\vec{p}^{\prime} s^{\prime}\right|\right\} \hat{O} \sum_{\vec{P}_{R}, S_{R}, \vec{p}, s}\left\{\left|\vec{P}_{R} S_{R}\right\rangle|\vec{p} s\rangle\right\}\right.\right. \\
& \times\left\{\left\langle\vec{P}_{R} S_{R}|\langle\vec{p} s|\} \mid P\right\rangle \delta\left(x-\frac{k^{\prime+}}{P^{+}}\right) \delta^{2}\left(\mathbf{k}_{\mathbf{T}}^{\prime}-\mathbf{k}_{\mathbf{T}}\right) .\right.
\end{aligned}
$$

Since later the nuclear matrix elements have to be evaluated by means of NR wave functions, the inserted states are normalized in a NR manner

$$
\left\langle\vec{P}^{\prime} \mid \vec{P}\right\rangle=(2 \pi)^{3} \delta^{3}\left(\vec{P}^{\prime}-\vec{P}\right),
$$

so that one has $|P\rangle=\sqrt{2 \overline{P_{0}}}|\vec{P}\rangle$ (cf. Equation (21))[23]. Because of this normalization and since, using IA and translational invariance,

$$
\begin{aligned}
\left\{\left\langle\vec{P}_{R} S_{R}|\langle\vec{p} s|\} \mid \vec{P} S\right\rangle=\right. & \left\langle\vec{P}_{R} S_{R}, \vec{p} s \mid \vec{P} S\right\rangle(2 \pi)^{3} \\
& \times \delta^{3}\left(\vec{P}-\vec{P}_{R}-\vec{p}\right) \delta_{S, S_{R}},
\end{aligned}
$$

Equation (25) can be rewritten as

$$
\begin{aligned}
f_{1}^{q, A}\left(x, \mathbf{k}_{\mathbf{T}}^{\mathbf{2}}\right)= & \frac{P_{0}}{P^{+}} \int d \vec{p} \sum_{S_{R}, s}\left\langle\vec{P} \mid \vec{P}_{R} S_{R}, \vec{p} s\right\rangle\left\langle\vec{P}_{R} S_{R}, \vec{p} s \mid \vec{P}\right\rangle \frac{1}{2 p_{0}} \\
& \times \int \frac{d k^{\prime+} \mathbf{d k}_{\mathbf{T}}^{\prime}}{2 k^{\prime+}(2 \pi)^{3}}\langle p s|\hat{O}| p s\rangle \\
& \times \delta\left(x-\frac{k^{\prime+}}{P^{+}}\right) \delta^{2}\left(\mathbf{k}_{\mathbf{T}}^{\prime}-\mathbf{k}_{\mathbf{T}}\right) .
\end{aligned}
$$

In the nuclear rest frame (RF), where $M_{A}=P_{0}=\sqrt{2} P^{+}$, introducing the Bjorken variable $x_{B}=\frac{Q^{2}}{2 M \nu}=\frac{M_{A}}{M} x$, and using the definition of $\alpha$, Eq. (14), one of the $\delta$ functions in the above equation can be written:

$$
\delta\left(x-\frac{k^{\prime+}}{P^{+}}\right)=\frac{1}{\alpha} \delta\left(\frac{x}{\alpha}-\frac{k^{\prime+}}{\alpha P^{+}}\right)=\frac{1}{\alpha} \frac{M_{A}}{M} \delta\left(\frac{x_{B}}{\alpha}-\frac{k^{\prime+}}{p^{+}}\right),
$$

so that Eq. (28), using the definition of the unpolarized nuclear spectral function, Eq. (18), and the expression of $f_{1}^{q, A}\left(x, \mathbf{k}_{\mathbf{T}}^{\mathbf{2}}\right)$ for a generic $A$ target, Eq. (22), becomes

$$
\begin{aligned}
f_{1}^{q, A}\left(x, \mathbf{k}_{\mathbf{T}}^{\mathbf{2}}\right)= & \frac{M_{A}}{M} \sum_{N} \int d E \int d \vec{p} P^{N}(\vec{p}, E) \frac{\sqrt{2} p^{+}}{p_{0}} \\
& \times \frac{1}{\alpha} f_{1}^{q, N}\left(\frac{x_{B}}{\alpha}, \mathbf{k}_{\mathbf{T}}^{\mathbf{2}}\right) .
\end{aligned}
$$

Equation (30) gives the $\mathbf{k}_{\mathbf{T}}$-dependent nuclear parton distribution in a convolutionlike form, in terms of the nuclear spectral function and of the $\mathbf{k}_{\mathbf{T}}$-dependent parton distribution for the internal, moving nucleon $N$. Similar expressions can be obtained for the other two nuclear $\mathbf{k}_{\mathbf{T}}$-dependent parton distributions, $h_{1}^{q, A}$ and $f_{1 T}^{\perp q, A}$, appearing in Eqs. (7) and (8).

Concerning the nuclear effects on the fragmentation functions, let us consider the standard unpolarized $\mathbf{k}_{\perp}$-dependent one, $D_{1}^{q, A, h}\left(z,\left(z \kappa_{\mathbf{T}}\right)^{2}\right)$. Since the hadron $h$ is produced by the fragmentation of a quark belonging to a nucleon with four-momentum $p$ in the nuclear medium, in an IA scenario, where the nucleon structure is not modified by the nuclear medium, $D_{1}^{q, A, h}$ is given by the corresponding quantity for the nucleon, with $z=\frac{p \cdot h}{p \cdot q}$, i.e.

$$
D_{1}^{q, A, h}\left(z, z^{2} \kappa_{\mathbf{T}}^{\mathbf{2}}\right)=D_{1}^{q, N, h}\left(\frac{p \cdot h}{p \cdot q},\left(\frac{p \cdot h}{p \cdot q}\right)^{2} \kappa_{\mathbf{T}}^{2}\right) .
$$

A similar expression can be obtained for the other nuclear $\mathbf{k}_{\mathbf{T}}$-dependent fragmentation function, $H_{1}^{\perp q, A, h}$, appearing in Eq. (7).

Because of the general structure of Eqs. (30) and (31), it is therefore possible to write the final formulas for the Sivers and Collins contributions to the single spin asymmetry of ${ }^{3} \mathrm{He}$, in I.A., in the nuclear R.F.

$$
A_{U T}^{\text {Collins }}=\frac{1-y}{1-y+y^{2} / 2}\left|\mathbf{S}_{\mathbf{T}}\right| \frac{N^{\text {Collins }}}{D},
$$




$$
A_{U T}^{\text {Sivers }}=\left|\mathbf{S}_{\mathbf{T}}\right| \frac{N^{\text {Sivers }}}{D},
$$

with

$$
\begin{aligned}
N^{\text {Collins }}= & \sum_{N} \int d E \int d \vec{p} \int d \alpha \delta\left(\alpha-\frac{\sqrt{2} p^{+}}{M}\right) P_{\perp}^{N}(\vec{p}, E) \\
& \times \frac{\sqrt{2} p^{+}}{p_{0}} \frac{1}{\alpha} \sum_{q} e_{q}^{2} \int d^{2} \kappa_{\mathbf{T}} d^{2} \mathbf{k}_{\mathbf{T}} \delta^{2}\left(\mathbf{k}_{\mathbf{T}}+\mathbf{q}_{\mathbf{T}}-\kappa_{\mathbf{T}}\right) \\
& \times \frac{\hat{\mathbf{h}} \cdot \boldsymbol{\kappa}_{\mathbf{T}}}{\mathbf{M}_{\mathbf{h}}} h_{1}^{q, N}\left(\frac{x_{B}}{\alpha}, \mathbf{k}_{\mathbf{T}}^{2}\right) H_{1}^{\perp q, N, h}\left(\frac{p \cdot h}{p \cdot q},\left(\frac{p \cdot h}{p \cdot q}\right)^{2} \kappa_{\mathbf{T}}^{2}\right),
\end{aligned}
$$

$$
\begin{aligned}
D= & \sum_{N} \int d E \int d \vec{p} \int d \alpha \delta\left(\alpha-\frac{\sqrt{2} p^{+}}{M}\right) P^{N}(\vec{p}, E) \frac{\sqrt{2} p^{+}}{p_{0}} \\
& \times \frac{1}{\alpha} \sum_{q} e_{q}^{2} \int d^{2} \kappa_{\mathbf{T}} d^{2} \mathbf{k}_{\mathbf{T}} \delta^{2}\left(\mathbf{k}_{\mathbf{T}}+\mathbf{q}_{\mathbf{T}}-\kappa_{\mathbf{T}}\right) \\
& \times f_{1}^{q, N}\left(\frac{x_{B}}{\alpha}, \mathbf{k}_{\mathbf{T}}^{\mathbf{2}}\right) D_{1}^{q, N, h}\left(\frac{p \cdot h}{p \cdot q},\left(\frac{p \cdot h}{p \cdot q}\right)^{2} \kappa_{\mathbf{T}}^{\mathbf{2}}\right)
\end{aligned}
$$

$$
\begin{aligned}
N^{\text {Sivers }}= & \sum_{N} \int d E \int d \vec{p} \int d \alpha \delta\left(\alpha-\frac{\sqrt{2} p^{+}}{M}\right) P_{\perp}^{N}(\vec{p}, E) \\
& \times \frac{\sqrt{2} p^{+}}{p_{0}} \frac{1}{\alpha} \sum_{q} e_{q}^{2} \int d^{2} \kappa_{\mathbf{T}} d^{2} \mathbf{k}_{\mathbf{T}} \delta^{2}\left(\mathbf{k}_{\mathbf{T}}+\mathbf{q}_{\mathbf{T}}-\kappa_{\mathbf{T}}\right) \\
& \times \frac{\hat{\mathbf{h}} \cdot \mathbf{k}_{\mathbf{T}}}{\mathbf{M}} f_{1 T}^{\perp q, N}\left(\frac{x_{B}}{\alpha}, \mathbf{k}_{\mathbf{T}}^{\mathbf{2}}\right) D_{1}^{q, N, h}\left(\frac{p \cdot h}{p \cdot q},\left(\frac{p \cdot h}{p \cdot q}\right)^{2} \kappa_{\mathbf{T}}^{2}\right) .
\end{aligned}
$$

I note in passing that the term $\frac{\sqrt{2} p^{+}}{p_{0}}$, appearing in Eqs. (34)-(36), is the so-called flux-factor [24,25], clearly emerging when one goes from field theoretical definitions in light-cone quantization to a NR framework, in order to use the available NR nuclear wave functions.

Equations (34)-(36) show that the Fermi motion and binding effects predicted by I.A. affect the longitudinal momentum in the parton distribution of the bound nucleon, and the longitudinal and transverse momentum in the fragmentation function of a struck quark belonging to a bound nucleon. The evaluation of Eqs. (32) and (33) is therefore rather involved and the extraction of the quantities of interest from experimental data looks quite cumbersome. Nevertheless, in the kinematics of the JLab experiments E-06-010 and E-06-011, everything becomes simpler. As a matter of facts, the chosen kinematics helps in two different ways. First of all, to favor pions from current fragmentation, the variable $z$ has been chosen to be around the value of 0.5 , which means that, in the kinematics of the proposed experiment, only ultrarelativistic pions with an energy $E_{h} \simeq p_{h} \simeq 2.4 \mathrm{GeV}$ are detected. Secondly, the pions are detected in a narrow cone around the direction of the momentum transfer $\vec{q}$. The maximum value of $\theta_{h q}$, the angle between the directions of the virtual photon and of the emitted pion is around $12^{\circ}$ [13]. Therefore, also the angle between $\vec{p}$ and $\vec{q}, \theta_{p q}$, and the one between $\vec{p}$ and $\vec{h}, \theta_{p h}$, are very similar. This makes possible to observe that, in DIS kinematics $(\nu \simeq|\vec{q}|)$

$$
\begin{aligned}
\frac{p \cdot h}{p \cdot q} \simeq & \frac{E_{h}\left(p_{0}-p \cos \theta_{p h}\right)}{\nu\left(p_{0}-p \cos \theta_{p q}\right)} \\
= & \frac{E_{h}}{\nu}\left\{1-\frac{p}{p_{0}}\left(\cos \theta_{p h}-\cos \theta_{p q}\right)+O\left[\left(\frac{p}{p_{0}}\right)^{2}\right]\right\} \\
= & \frac{E_{h}}{\nu}\left\{1-\frac{p}{p_{0}} \sin \left(\frac{\theta_{p h}+\theta_{p q}}{2}\right) \sin \left(\frac{\theta_{p q}-\theta_{p h}}{2}\right)\right. \\
& \left.+O\left[\left(\frac{p}{p_{0}}\right)^{2}\right]\right\} \\
\simeq & \frac{E_{h}}{\nu}=z,
\end{aligned}
$$

the last identity holding due to the little difference between $\theta_{p q}$ and $\theta_{p h}$. As a matter of facts, the leading nuclear effects discussed here, affecting the nuclear parton distributions, are of the order $O\left(\frac{p}{p_{0}}\right)$, and therefore the $p$-dependence in the argument of the fragmentation functions, given by $p / p_{0}$ multiplied by a vanishing coefficient, yields subleading effects which can be disregarded.

Eventually, inserting Eq. (37) in Eqs. (34)-(36), Eqs. (32) and (33) reduce to Eqs. (9) and (10), which are therefore the expressions to be evaluated in the kinematics of the JLab experiments.

Applying the proposed formalism, the SSAs for ${ }^{3} \mathrm{He}$ can be estimated by calculating Eqs. (9) and (10), i.e. by modelling the functions $G_{\perp}^{3, N}(\alpha)$ and $F^{3, N}(\alpha)$, dependent on the nuclear structure, and the five functions $f_{1}^{q, N}, h_{1}^{q, N}$, $f_{1 T}^{\perp q, N}, D_{1}^{q, h}, H_{1}^{\perp q, h}$, depending on the nucleon structure. The nuclear part can be properly treated by using a realistic nuclear polarized spectral function, Eq. (19), describing the Fermi motion and binding effects, which allows one to evaluate the light-cone momentum distributions, $G_{\perp}^{3, N}(\alpha)$ and $F^{3, N}(\alpha)$, according to Eqs. (15)-(18). The nucleon part can be evaluated by using fits of experimental data, whenever they are available, or results of model calculations. The ingredients used in the present analysis will be discussed in the next section.

Now the main issue of extracting the neutron information from the nuclear asymmetries will be addressed. To this aim, in $[13,14]$, it has been proposed to use a method, the one suggested and justified, for the first time for DIS processes, in [12]. In that paper, it was shown that in polarized DIS nuclear effects described by IA, i.e., Fermi motion and binding effects, are safely taken care of by considering the nucleons with an effective polarization, safely predictable with realistic nuclear wave functions [11]. In the present case, the idea leads to approximate the experimental asymmetry for ${ }^{3} \mathrm{He}, A_{3}^{\exp }$, by the follow- 
ing expression:

$$
A_{3}^{\mathrm{exp}, i} \simeq 2 f_{p} p_{p} A_{\vec{p}}^{i}+f_{n} p_{n} A_{\vec{n}}^{i},
$$

where $f_{p(n)}$ is the proton (neutron) "dilution factor", the index $i$ means Collins or Sivers, $A_{\vec{p}(\vec{n})}^{i}$ is the proton (neutron) asymmetry and the effective nucleon polarizations are

$$
\begin{gathered}
p_{p}=\int d E \int d \vec{p} P_{\perp}^{p}(\vec{p} E)=-0.028 \pm 0.004, \\
p_{n}=\int d E \int d \vec{p} P_{\perp}^{n}(\vec{p} E)=0.86 \pm 0.02,
\end{gathered}
$$

as obtained by averaging many few-body calculations, available at the time of publication of Refs. [11,26]. One should notice that, were polarized ${ }^{3} \mathrm{He}$ a perfect effective neutron target with all the nucleons in $S$ wave, one would find $p_{n}=1$ and $p_{p}=0$.

Eventually, the dilution factors are

$$
f_{p(n)}(x, z)=\frac{\sum_{q} e_{q}^{2} f_{1}^{q, p(n)}(x) D_{1}^{q, h}(z)}{\sum_{N=p, n} \sum_{q} e_{q}^{2} f_{1}^{q, N}(x) D_{1}^{q, h}(z)} .
$$

While the approximation Eq. (38), theoretically proposed and justified in [12], has been widely used in the treatment of DIS data, the possibility of using it also in SIDIS deserves a careful analysis, which will be performed in the next section. Since now it is worth to stress that, if Eq. (38) were a good approximation of reality, it would be possible to use it to extract the neutron Asymmetry according to the following formula, suggested in [12]:

$$
A_{n}^{i} \simeq \frac{1}{p_{n} f_{n}}\left(A_{3}^{\mathrm{exp}, i}-2 p_{p} f_{p} A_{p}^{\mathrm{exp}, i}\right) \text {. }
$$

If one uses in Eq. (42) a realistic calculation to simulate the experimental data $A_{3}^{\text {exp, } i}$, and the model used for the proton in the calculation to simulate the experimental data for $A_{p}^{\text {exp }, i}$, if nuclear effects were safely taken care of by Eq. (38), one should be able to extract, according to Eq. (42), the neutron asymmetry used as an input for the calculation. In the same way, the neutron information would be obtained safely from nuclear and proton data using Eq. (42), if the main nuclear structure effects were properly described by Eq. (38).

\section{RESULTS AND DISCUSSION}

As stated in the previous section, in order to calculate the SSAs of ${ }^{3} \mathrm{He}$, Eqs. (9) and (10), one nuclear structure ingredient, the polarized spectral function, Eq. (19), providing the light-cone momentum distributions $G_{\perp}^{3, N}(\alpha)$ and $F^{3, N}(\alpha)$, and five nucleon structure ingredients, the functions $f_{1}^{q, N}, h_{1}^{q, N}, f_{1 T}^{\perp q, N}, D_{1}^{q, h}, H_{1}^{\perp q, h}$, have to be modeled.
In the following, the quantities used in the calculation are presented and discussed.

Concerning the nuclear part, the spin-dependent polarized spectral function, Eq. (19), corresponding to the Argonne V18 interaction (AV18, [27]), has been evaluated. This has been done along the line of Ref. [28], using the overlaps calculated in [29] by means of a wave function obtained by the method of Ref. [30]. The procedure gives, for the proton and the neutron in ${ }^{3} \mathrm{He}$, the effective polarizations $p_{p}=-0.024$ and $p_{n}=0.878$, respectively, in agreement with the analysis of Ref. [11], Eqs. (39) and (40), and only slightly different from the predictions of the AV14 interaction $\left(p_{p}=-0.026, p_{n}=0.873\right.$ [28]), as expected.

Concerning the nucleon, to perform the calculation, the following five ingredients have been used:

(1) for the unpolarized $\mathbf{k}_{\mathbf{T}}$-dependent parton distribution, $f_{1}^{q, N}\left(x, \mathbf{k}_{\mathbf{T}}^{2}\right)$, use has been made of the usual Gaussian factorization:

$$
f_{1}^{q, N}\left(x, \mathbf{k}_{\mathbf{T}}^{2}\right)=f_{1}^{q, N}(x) G\left(\mathbf{k}_{\mathbf{T}}^{2}\right),
$$

with [31]

$$
G\left(\mathbf{k}_{\mathbf{T}}^{2}\right)=\frac{1}{\pi\left\langle\mathbf{k}_{\mathbf{T}}^{2}\right\rangle} e^{-\mathbf{k}_{\mathbf{T}}^{2} /\left\langle\mathbf{k}_{\mathbf{T}}^{2}\right\rangle},
$$

where $\left\langle\mathbf{k}_{\mathbf{T}}^{2}\right\rangle=0.25 \mathrm{GeV}^{2}$, and for the standard parton distribution $f_{1}^{q, N}(x)$ use has been made of the parameterization of Ref. [32];

(2) for the transversity distribution, $h_{1}^{q, N}$, still experimentally poorly known, use has been made of the same Gaussian factorization above, with the ansatz $h_{1}^{q}(x)=g_{1}^{q}(x)$, i.e., the transversity distribution has been taken to be equal to the standard helicity distribution. This gives certainly the correct order of magnitude. In particular, the parameterization of Ref. [33] has been used;

(3) for the Sivers function, $f_{1 T}^{\perp q}\left(x, \mathbf{k}_{\mathbf{T}}^{\mathbf{2}}\right)$ in Eq. (10), use has been made of a fit of the few available data, proposed in Ref. [31];

(4) for the unpolarized fragmentation function $D_{1}^{q, h}\left(z,\left(z \kappa_{\mathbf{T}}\right)^{2}\right)$, different models are used for evaluating the Sivers and Collins Asymmetries. For evaluating the Sivers one, use has been made of the Gaussian factorization:

$$
D_{1}^{q, h}\left(z,\left(z \kappa_{\mathbf{T}}\right)^{2}\right)=D_{1}^{q}(z) G\left[\left(z \kappa_{\mathbf{T}}\right)^{2}\right],
$$

with [31]

$$
G\left(\mathbf{p}_{\mathbf{T}}^{2}\right)=\frac{1}{\pi\left\langle\mathbf{p}_{\mathbf{T}}^{2}\right\rangle} e^{-\mathbf{p}_{\mathbf{T}}^{2} /\left\langle\mathbf{p}_{\mathbf{T}}^{2}\right\rangle},
$$

where $\left\langle\mathbf{p}_{\mathbf{T}}^{2}\right\rangle=0.2 \mathrm{GeV}^{2}$, and using for the standard fragmentation function $D_{1}^{q}(z)$ the parameterization in Ref. [34]. For evaluating the Collins Asymmetry, use has been made of the model calculation of 
$D_{1}^{q, h}\left(z,\left(z \kappa_{\mathrm{T}}\right)^{2}\right)$ discussed in Ref. [35], in particular, the one performed considering pseudoscalar pionquark coupling, Eq. (6) of that paper. The latter choice has been done for consistency reasons, since the same scenario has been used to model the Collins fragmentation function in evaluating the Collins Asymmetry, as explained in the following point;

(5) for the basically unknown Collins fragmentation function, $H_{1}^{\perp q}\left(z,\left(z \kappa_{\mathbf{T}}\right)^{2}\right)$, appearing in Eq. (9), use has been made of the model calculation of Ref. [35], in particular, of the contribution arising from pion loops, considering pseudoscalar pion-quark coupling, Eqs. (8)-(12) of that paper.

To fix the ideas, results will be shown for the production of $\pi^{-}$, although everything can be immediately extended to $\pi^{+}$production.

The results for the SSAs of ${ }^{3} \mathrm{He}$ are not very illuminating by themselves, being the calculation strongly dependent on the models used as an input for the nucleon transverse spin structure, i.e. on the models chosen for the basically unknown $h_{1}$ distribution, Sivers distribution and Collins fragmentation function. In presenting the results, it is better therefore to emphasize the relevance of nuclear effects in the extraction of the neutron information. A convenient scheme to illustrate them is the following. One could assume that the full calculation, i.e. the evaluation of Eqs. (9) and (10), using the ingredients listed above, represents a set of experimental data. The neutron information is then extracted from them by using different models of the ${ }^{3} \mathrm{He}$ spin structure. The obtained result is then compared to the neutron SSA used as an input in the calculation. The closer the obtained curves are, the better the proposed extraction procedure works. This would allow to proper take into account Fermi motion and binding effects in obtaining the neutron information, without performing difficult deconvolutions of the Eqs. (9) and (10), once the data are available. Of course this procedure does not take into account effects beyond Fermi motion and binding, i.e. beyond IA. This scheme is used to present the results in Figs. 2 and 3. The free neutron asymmetry used as a model in the calculation, given by a full line, is compared with two curves. One is the quantity

$$
\bar{A}_{n}^{i} \simeq \frac{1}{f_{n}} A_{3}^{\mathrm{exp}, i},
$$

where $A_{3}^{\text {exp, } i}$ is the result of the full calculation, simulating data, and $f_{n}$ is the neutron dilution factor, Eq. (41). Equation (47) is the relation one would expect to hold between the ${ }^{3} \mathrm{He}$ and the neutron SSAs if there were no nuclear effects, i.e. ${ }^{3} \mathrm{He}$ were a system of free nuleons in a pure $S$ wave. In fact it can be obtained from Eq. (42) by imposing $p_{n}=1$ and $p_{p}=0 . \bar{A}_{n}^{i}$ is given by the dotted curve in the figures. The third curve, the dashed one, is obtained by using Eq. (42), i.e. thinking to ${ }^{3} \mathrm{He}$ as a nucleus where the effects of its complicated spin structure, leading to a depolarization of the bound neutron, together with the ones of Fermi motion and binding, can be easily taken care of, according to Eq. (38), by parameterizing the nucleon effective polarizations of ${ }^{3} \mathrm{He}$. For the latter quantities, in Eq. (42) the values given in Eqs. (39) and (40) from Ref. [11], not the ones obtained by the AV18 interaction used in the full calculation, have been chosen. This is done
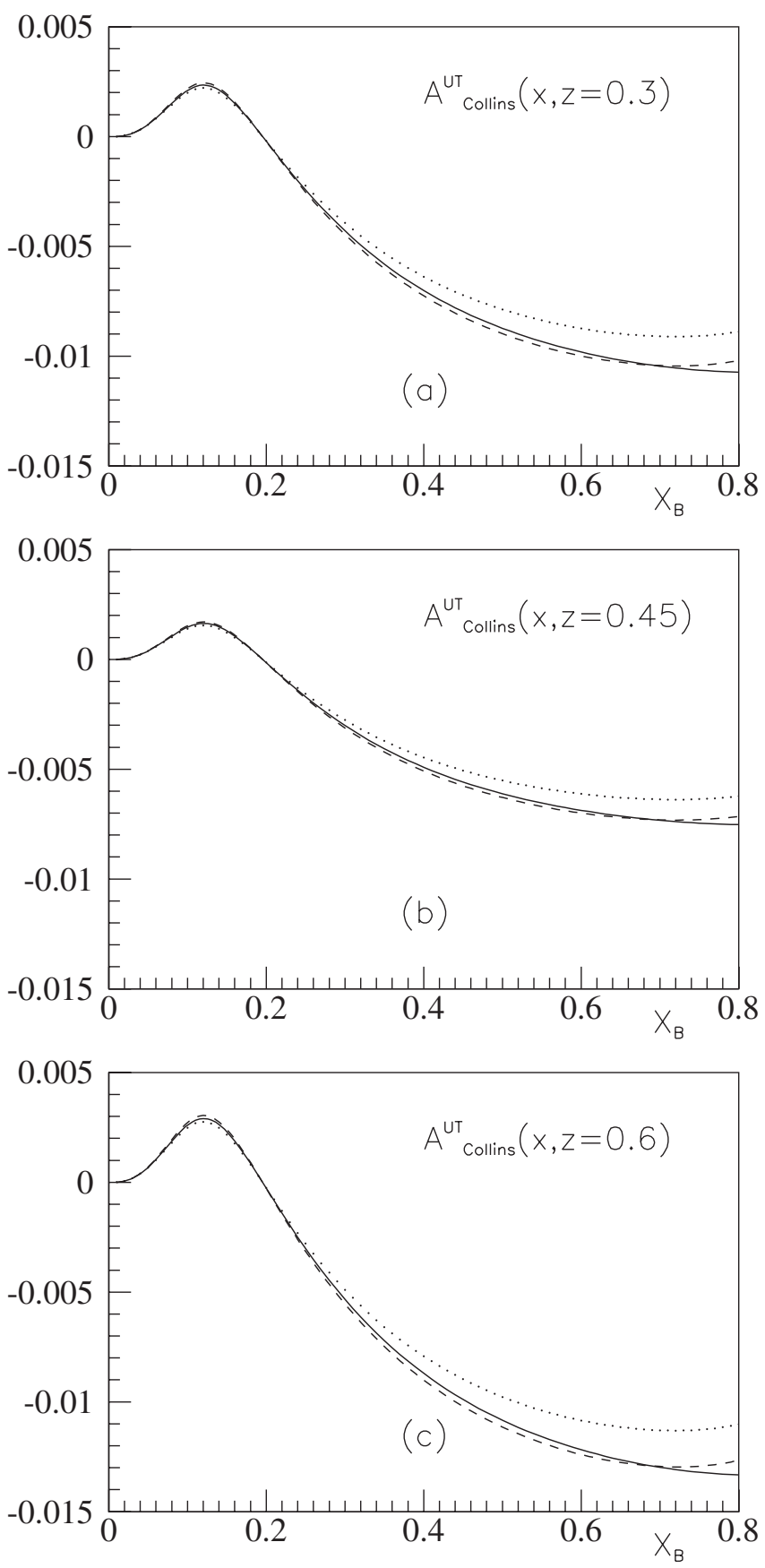

FIG. 2. The model neutron Collins asymmetry for the production of $\pi^{-}$(full), and the one extracted from the full calculation according to Eq. (42) (dashed) and Eq. (47) (dotted), for $z=0.3$, $0.45,0.6$, in panels (a), (b), (c), respectively, for $Q^{2}=2.2 \mathrm{GeV}^{2}$. 

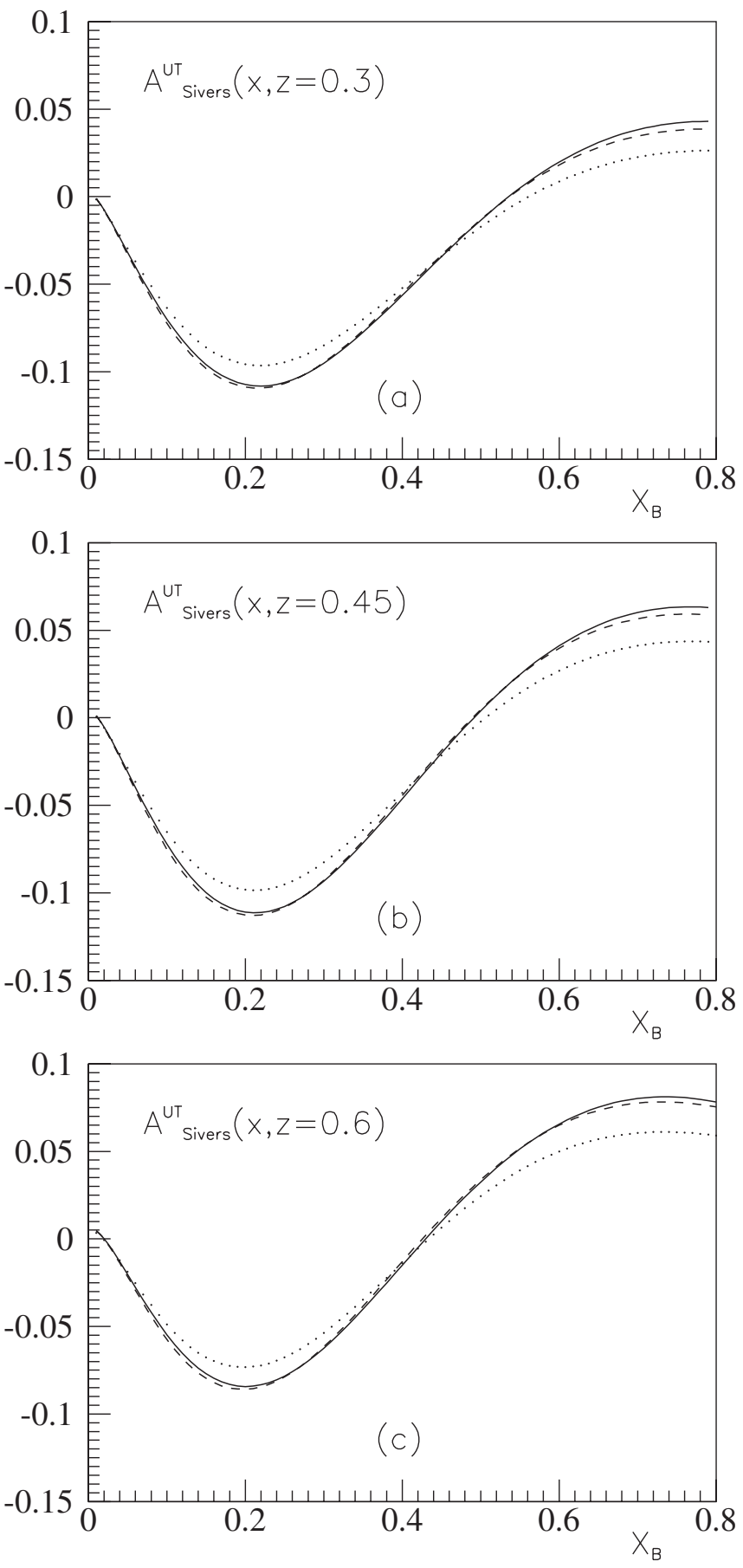

FIG. 3. The same as in Fig. 2, but for the Sivers asymmetry.

to take into account the possible error due to the choice of the nucleon-nucleon potential.

In Fig. 2 it is shown the result of the procedure for the Collins Asymmetry, corresponding to the production of $\pi^{-}$, as a function of $x_{B}$ and for various values of $z$. The values $Q^{2}=2.2 \mathrm{GeV}^{2}$ and $\theta_{q h}=10^{\circ}$, central values in the kinematics of the TJNAF experiment [13], have been chosen for the calculation. In the latter experiment, data will be taken for $0.13<x_{B}<0.41,0.46<z<0.59$ and $E_{\mathrm{lab}}=6 \mathrm{GeV}$. It is clear from the figure that the difference between the full and dotted curve, showing the amount of nuclear effects, is sizable, being around $10-15 \%$ for any $x$ and $z$ values in the range relevant for the planned experiment, while the difference between the dashed and full curves reduces drastically to a few percent, showing that the extraction scheme Eq. (42) takes safely into account the spin structure of ${ }^{3} \mathrm{He}$, together with Fermi motion and binding effects. One should remember that in the proposed experiments only the region $0.13<x_{B}<0.41$ will be actually explored.

In Fig. 3 the same is shown for the Sivers Asymmetry, again in the kinematics of the proposed TJNAF experiment. In this region, for the Sivers Asymmetry, the same conclusions discussed previously for the Collins one hold. I reiterate that one should not take the absolute size of the Asymmetries too seriously, being the obtained neutron Collins and Sivers asymmetry strongly dependent on the models chosen for the unknown distribution functions. One should instead consider the difference between the curves which are shown, a model independent feature which is the most relevant outcome of the present investigation.

From Figs. 2 and 3 it is clear therefore that Eq. (42) will be a valuable tool for the data analysis of the experiments $[13,14]$.

In principle, effects beyond IA could be relevant in the process under scrutiny. Among them, shadowing effects and final state interactions (FSI) could play a role. As a matter of facts, some doubts on the possibility of using safely the extraction procedure Eq. (42) due to shadowing effects have arisen [36]. One should remember anyway that these effects are supposed to be active for values of Bjorken $x$ smaller than the ones explored by the planned experiments. From this point of view, problems should not arise. Concerning FSI, the assumption that even the outgoing hadron, which can be slow, does not interact further, makes the use of the IA in SIDIS more questionable with respect to the standard inclusive DIS case. Moreover, since the planned values of $Q^{2}$ are smaller than $3 \mathrm{GeV}^{2}$, in principle the effects of FSI cannot be ignored. One should notice anyway that, in the planned experiments, the energy of the emitted pions is high, being $0.46<z<0.59$, chosen to give $E^{\pi} \simeq 2.4 \mathrm{GeV}$. The latter observation should make rather safe the use of IA results for the analysis of the JLab experiments. Moreover, in the numerator of the asymmetry, Eq. (1), the effects of any spin-independent interaction should cancel out, in particular, for a spinless hadron as the pion. In any case, the analysis of FSI for this complicated process deserves a dedicated analysis. This work is in progress, together with the inclusion in the scheme of other more realistic models of the nucleon structure, able to predict reasonable figures for the experiments.

\section{CONCLUSIONS}

The recent measurement of the single spin asymmetry for the proton and the deuteron exhibited the importance of 
safely accessing the same quantity for the neutron. As for any polarized neutron observable, ${ }^{3} \mathrm{He}$, due to its peculiar spin structure, is the natural target. Two experiments, aimed at measuring azimuthal asymmetries in the production of $\pi^{ \pm}$from transversely polarized ${ }^{3} \mathrm{He}$, have been approved at JLab. It is planned to obtain the neutron information from ${ }^{3} \mathrm{He}$ data, using a procedure that performs well in the inclusive DIS case, being able to take care of several crucial aspects of nuclear dynamics, such as the spin structure of ${ }^{3} \mathrm{He}$ and the momentum and energy distributions of bound nucleons. Here, the problem whether or not that procedure can be extended to the present SIDIS scenario, involving therefore fragmentation functions and not only parton distributions, has been thoroughly analyzed, in impulse approximation. The general formulas including the nuclear structure effects are derived, and evaluated in the specific JLab kinematics, using the AV18 interaction to describe the nuclear structure and parametrizations of data or suitable model calculations to describe the nucleon part. The initial transverse momentum of the struck quark has been properly included in the calculation. It is found that, in the kinematics of the proposed experiments, Fermi motion and binding effects can be safely taken care of by a simple extraction procedure, where the only nuclear structure ingredients are the nucleon effective polarizations, quantities known from precise few-body calculations in a rather model independent way. The possible role of final state interactions, and the evaluation of the quantities of interest by means of realistic models of the nucleon, to get figures for the planned experiments, have been addressed and are under investigation.

\section{ACKNOWLEDGMENTS}

I am grateful to S. Noguera and V. Vento for fruitful discussions, and to the Department of Theoretical Physics of the Valencia University, where this study was mainly done, for warm hospitality. This work is supported in part by the INFN-CICYT agreement and by the Generalitat Valenciana under the contract No. AINV06/118.
[1] A. Barone, A. Drago, and P. Ratcliffe, Phys. Rep. 359, 1 (2002).

[2] Proceedings of the International Workshop on Transverse Polarisation Phenomena in Hard Processes (Transversity 2005), Como, Italy, 2005, edited by P. Ratcliffe.

[3] J. C. Collins, Nucl. Phys. B396, 161 (1993).

[4] D. Sivers, Phys. Rev. D 41, 83 (1990); 43, 261 (1991).

[5] P. J. Mulders and R. D. Tangerman, Nucl. Phys. B461, 197 (1996); B484, 538(E) (1997).

[6] A. M. Kotzinian and P. J. Mulders, Phys. Lett. B 406, 373 (1997).

[7] D. Boer and P. J. Mulders, Phys. Rev. D 57, 5780 (1998).

[8] A. Airapetian et al. (HERMES Collaboration), Phys. Rev. Lett. 94, 012002 (2005).

[9] V. Y. Alexakhin et al. (COMPASS Collaboration), Phys. Rev. Lett. 94, 202002 (2005).

[10] S. J. Brodsky and S. Gardner, Phys. Lett. B 643, 22 (2006).

[11] J. L. Friar, B. F. Gibson, G. L. Payne, A. M. Bernstein, and T. E. Chupp, Phys. Rev. C 42, 2310 (1990).

[12] C. Ciofi degli Atti, S. Scopetta, E. Pace, and G. Salmè, Phys. Rev. C 48, R968 (1993).

[13] J.-P. Chen and J.-C. Peng, Spokespersons JLab-PAC29 Proposal No. E-06-010.

[14] E. Cisbani and H. Gao, Spokespersons JLab-PAC29 Proposal No. E-06-011.

[15] A. Bacchetta et al., Phys. Rev. D 70, 117504 (2004).

[16] Here and in the following, the light-cone variables are defined as $a^{ \pm}=\frac{1}{\sqrt{2}}\left(a_{0} \pm a_{3}\right)$.

[17] C. Ciofi degli Atti, E. Pace, and G. Salmè, Phys. Rev. C 46, R1591 (1992); 51, 1108 (1995).

[18] R. L. Jaffe, hep-ph/9602236.

[19] A. Bacchetta, Ph.D. Thesis, Vrije Universiteit, 2002.

[20] R. W. Schulze and P. U. Sauer, Phys. Rev. C 48, 38 (1993);
56, 2293 (1997).

[21] F. Bissey, V. Guzey, M. I. Strikman, and A. W. Thomas, Phys. Rev. C 65, 064317 (2002).

[22] S. Scopetta, Phys. Rev. C 70, 015205 (2004).

[23] P. J. Mulders, Phys. Rep. 185, 83 (1990).

[24] D. Kusno and M. J. Moravcsik, Phys. Rev. D 20, 2734 (1979).

[25] L. L. Frankfurt and M. I. Strikman, Phys. Rep. 160, 235 (1988).

[26] Since that time, other calculations have been performed. For example, in Table II of Ref. [28], some values of effective polarizations obtained by considering also the Coulomb repulsion between the protons in ${ }^{3} \mathrm{He}$, not included in the analysis of Ref. [11], are reported.

[27] R. B. Wiringa, V. G. J. Stocks, and R. Schiavilla, Phys. Rev. C 51, 38 (1995).

[28] A. Kievsky, E. Pace, G. Salmè, and M. Viviani, Phys. Rev. C 56, 64 (1997).

[29] E. Pace, G. Salmè, S. Scopetta, and A. Kievsky, Phys. Rev. C 64, 055203 (2001).

[30] A. Kievsky, M. Viviani, and S. Rosati, Nucl. Phys. A 577, 511 (1994).

[31] M. Anselmino et al., Phys. Rev. D 72, 094007 (2005).

[32] M. Glück, E. Reya, and A. Vogt, Eur. Phys. J. C 5, 461 (1998).

[33] M. Glück, E. Reya, M. Stratmann, and W. Vogelsang, Phys. Rev. D 63, 094005 (2001).

[34] S. Kretzer, Phys. Rev. D 62, 054001 (2000).

[35] D. Amrath, A. Bacchetta, and A. Metz, Phys. Rev. D 71, 114018 (2005).

[36] L. Frankfurt, V. Guzey, and M. Strikman, Phys. Lett. B 381, 379 (1996). 